\title{
Design and implementation of the system to support making medical decisions aid to the population
}

\author{
Ekaterina Stodelova ${ }^{1}$ and Galina Korableva ${ }^{2, *}$ \\ ${ }^{1}$ K. G. Razumovsky Moscow State University of Technologies and Management (Smolensk branch), \\ Lenin street 77, Smolensk region, Vyazma, 215100, Russia
}

\begin{abstract}
The publication discusses the issues and results of designing an automated system for supporting medical decision-making on issues of antirabic prevention and aid to the population. The need to provide medical care in the framework of preventing deaths from rabies infection and carrying out preventive measures to prevent such infections is relevant not only in the Russian Federation, but also in many countries of the world. Algorithms for providing medical care and carrying out rabies vaccination are complex, requiring certain professional skills from medical professionals, as well as care and accuracy from patients. Therefore, in manual mode, they are time-consuming both in terms of registering information about the treatment performed, and in terms of the speed of forming medical decisions when providing medical care or when correcting violations of previously defined algorithms. For computer support of these processes, an automated system for supporting medical decision-making has been developed using the tools of the Clarion 10 relational database management system. For various categories of patients and citizens undergoing rabies prevention, experts have developed and entered into the database templates of treatment courses, which are assigned by the system after analyzing the clinical data of patients and the initial or repeated fact of their request for help. These templates and algorithms for analyzing the possibilities of their application are similar to products that allow you to link assumptions and conclusions when making decisions. The automated medical decision support system allows you to register patients and persons undergoing rabies prevention, assign them treatment courses and vaccination schemes, adjust treatment and prevention methods in case of violations of previously prescribed ones, and generate statistical reports. The developed software product received the author's certificate no. 2018663452 dated 26.10.2018 from the Federal service for intellectual property (Rospatent). The software product has been tested in first aid rooms in Moscow, in the practice of the polyclinic department of the First City hospital named after N. I. Pirogov.
\end{abstract}

\section{Introduction}

\footnotetext{
*Corresponding author: gkorableva2008@yandex.ru
} 
Rabies is a dangerous viral disease of animals and humans, characterized by signs of polio and absolute lethality. It is found in more than 150 countries. The only treatment for infection is a timely course of vaccination. WHO and the Global Alliance against rabies have established the partnership "United against rabies» to develop a worldwide strategy to combat rabies-related deaths.

According to Rospotrebnadzor in 2019, more than 400 thousand people appealed to medical institutions for assistance from received animal bites, about 100 thousand of them are children under 14 years of age. According to the results of medical and preventive measures in 2019, only two fatal cases of people from rabies were recorded in Moscow and the Amur region [1-10].

In 2019, an increase in the number of farm animals infected with rabies was also detected in the Moscow, Tver, Ryazan, Kursk, Bryansk, Chelyabinsk, Sverdlovsk, Voronezh, Lipetsk regions, Krasnoyarsk territory and Buryatia [9].

The rabies virus multiplies in the salivary glands of animals (foxes, wolves, dogs, cats, bats, rodents, livestock). Human infection can occur both when animals bite, and when the skin and mucous membranes are salivated. A person's susceptibility to infection depends on the location and severity of the injury: the closer to the head and neck and deeper the wound is, the higher the probability of the disease is.

Once in the human body, the virus spreads through the perineural spaces. The virus propagates mainly in the spinal cord and brain [8].

Diagnosis of the disease is based on clinical manifestations and epidemiological history. Lifetime isolation of the virus from the patient is difficult in clinical practice. A diagnostic sign suggesting the presence of the rabies virus is the death of the animal after 6-7 days. The treatment is specific anti-rabies prophylaxis with vaccine and serum.

Not only owners of cats and dogs, or farm animals and their owners, but also all citizens who may encounter stray animals living in the basements of houses, on the territories adjacent to them, can become victims of the bite of animals with rabies.

The Resolution of the Chief state sanitary doctor of the Russian Federation as of May 6, 2010 N 54 Moscow "Approval of SR 3.1.7.2627-10" defines the categories of employees who need rabies vaccination for their professional activities in order to reduce the risk of their infection:

- employees of services that capture animals (catchers, drivers, hunters, foresters, and others),

- employees of veterinary stations for the control of animal diseases who have contact with animals (veterinarians, paramedics, laboratory assistants, junior staff),

- employees of research institutes and diagnostic laboratories performing research on rabies,

- employees of vivariums and other institutions that work with animals,

- persons with a high risk of infection (pathologists, specialists involved in parenteral treatment of patients with rabies), working in medical institutions of preventive vaccination against rabies [1-4].

Assistance to people who have been bitten by animals begins on the day of their appeal and depends on a variety of conditions that must be taken into account when carrying out vaccination. These conditions include - the first or repeated bite for a short time, the place of the bite, the degree of damage, the day of seeking help. A traumatologist-orthopedist or other medical professional must take into account all these conditions and determine the algorithm of medical care and vaccination scheme for the injured person.

This algorithm includes the necessary type of vaccine and the scheme of its introduction to the patient. Moreover, this process is long in time, requires the further presence of the patient on certain days for vaccination, in case of violation of the previously defined algorithm by the doctor, it is necessary to adjust it using a non-standard method. In the 
presence of a large number of such patients, the process is time-consuming and requires precision in conducting preventive procedures.

Therefore, an automated decision support system for providing anti-rabis care and prevention has been developed.

The analysis of the Russian software market has shown that there are no software products for this purpose, including in medical institutions that provide anti-rabies care and prevention.

The purpose of this study is to develop a basic algorithm of functioning of the automated system to support medical decision-making (AMDSS) on rabies prevention and assistance.

\section{Materials and Methods}

As research methods, we used a systematic approach to the study of the subject area "antirabies care and prevention", methods of analysis and synthesis.

The automated decision support system was developed using the database management system (DBMS) and Clarion 10 programming language of the same name. This DBMS includes powerful tools for implementing the RAD approach to application development and is oriented towards a relational data model. The AMDS database includes the necessary information for solution generating and allows you to register user actions at different stages of treatment and prevention procedures within the generated solutions.

The AMDS database includes 19 relational tables, 10 of which are reference tables. When designing the AMDSS database, the normal forms method is used.

The reference tables of the database contain conditional-permanent information entered at the stage of setting up the automated system, it includes reference lists: doctors, organizations, insurance companies, medical specialties, types of activities, medications, injuries, body parts, categories of contact with animals, types of preventive measures.

The main information necessary for the work of doctors in the database of the automated decision support system is stored in the register of bites, the register of procedures after bites, the register of prevention facts for employees of organizations, the register of preventive procedures, and treatment course templates.

A structural approach is used to develop algorithms that implement AMDSS functions. Functional IDEF0 (SADT) models of four hierarchical levels are constructed, which allow to determine the main algorithms for forming medical decisions and their subsequent correction if necessary.

All the processes of forming medical decisions are implemented on the basis of a production model of knowledge, allowing you to analyze the initial information for decision - making, to choose the right template for the course of treatment or to determine the algorithm of actions of the doctor.

AMDSS performs the following functions:

- registration of patients who have applied for anti-rabis treatment and their existing injuries,

- analysis of the patient's condition and injuries, as well as the further automated selection of the scheme of medical measures for patients who have applied for anti-rabis help, including for cases of the repeated appeal,

- registration of persons who undergo anti-rabis prophylaxis,

- automated formation of an anti-rabis prophylaxis scheme,

- determination by means of the software product of the most critical category of damage to the patient, even in cases of multiple bites done by animals,

- formation of medical decisions to adjust or cancel previously formed schedules of medical procedures for patients who have applied for anti-rabies care, if the patient was not able to receive the necessary medical measures at the appointed time,

- registration of medical or preventive measures performed for specific patients using 
software tools,

- storing information about patients who received anti-rabies care in a particular medical institution in an electronic archive with the organization of rapid sampling of information,

- the ability to create AMDSS user schemes for conducting medical measures in the treatment of patients who have requested anti-rabis help, and / or prevention of such cases,

- flexible configuration of AMDSS for a specific medical base.

The AMDSS structure includes the following functional elements: a database, a DBMS, a database of rules for generating solutions implemented by using the Clarion programming language, a subsystem for selecting, canceling or correcting treatment schemes, and an interface subsystem (figure 1).

\section{Results and discussions}

During the research, we formalized the studied materials on the treatment and prevention of rabies and developed a generalized algorithm for the functioning of AMDSS, implemented in its software modules using the Clarion DBMS.

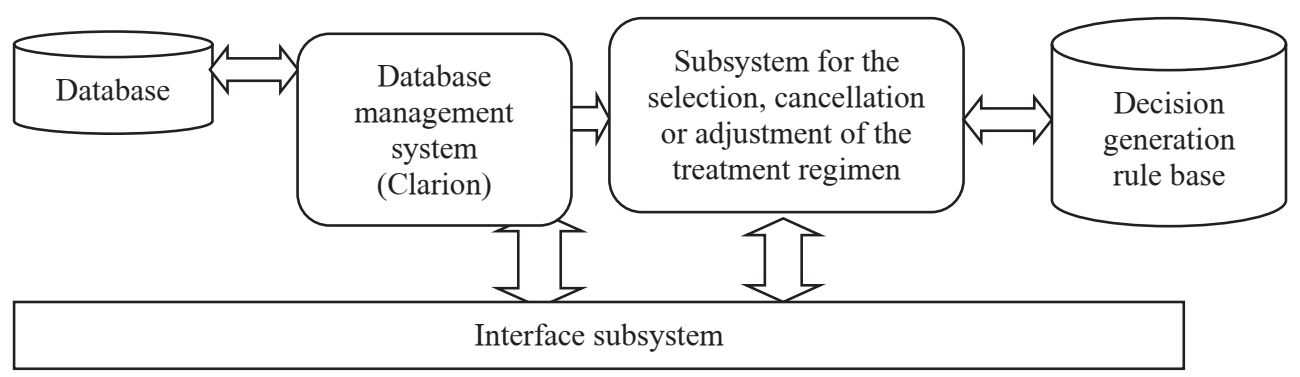

Fig. 1. Structure of the AMDSS.

The algorithm of functioning of AMDSS reflects the method of working with the software product and includes the following main actions.

1) First, you need to configure the software product for a specific medical institution and the subject area "Anti-rabies care and prevention". To do this, the user must enter the information in the directories of the database "Doctors", "Specializations/ specialties of the doctors", "Types of medical/preventive measures", "Vaccines, drugs", "Types of animals", "Categories", "Types of injuries", "Body Parts", "Insurance companies", "Organizations that apply for anti-rabies prevention to their employees".

2) An expert or an experienced doctor introduces treatment or prevention schemes for AMDSS users in the form of treatment course templates and generates rules for generating solutions.

3) After that, you can work with AMDSS in one of the two selected modes of operation: "Anti-rabies prevention" or "Anti-Rabies help".

4) When selecting the "Anti-Rabies prevention" mode of operation, enter the data about persons undergoing prophylaxis: last name, first name, patronymic, date of birth, gender, weight, insurance number, the insurance company where the insurance was issued, and the organization where the person works.

5) Based on the selected prophylaxis schemes for people who are directed to anti-rabies prophylaxis, and on the data entered in the log of prevention facts for employees of organizations, AMDSS tools are used to create a schedule of prophylactic measures. After filling in the personal data about the persons sent for prophylaxis, the corresponding AMDSS module is called from the log interface by clicking the "Prevention Measures" button, which 
generates a list of measures for the entered employee or a list of employees by the "Fill in" command.

6) After each performed prophylactic anti-rabies activity, the doctor makes a note in the register of preventive procedures, forming an entry for a specific person. If a person fails to appear on the appointed date for the next preventive anti-rabies activity, the doctor uses the software to adjust the previously drawn up plan of measures or makes a new one.

7) When selecting the "Anti-Rabies help" mode, the data about the patient who requested help and the data about the received injuries are entered in the bite log (figure 2).

8) After entering the data, the AMDSS software analyzes the injuries received by the patient and forms a conclusion about the category of depth of contact with the animal, especially in the case of multiple heterogeneous injuries.

9) After this, the module for generating measures on received bites is called, which checks whether the patient was previously registered in the bite log or in the register of anti-rabies prevention facts.

10) Depending on the information found in the AMDSS database and based on the category of the depth of contact between the patient and the animal, in accordance with the instructions for organizing medical and preventive work on population immunization against rabies [5], the treatment scheme and schedule of treatment measures are formed by means of AMDSS by calling the appropriate module.

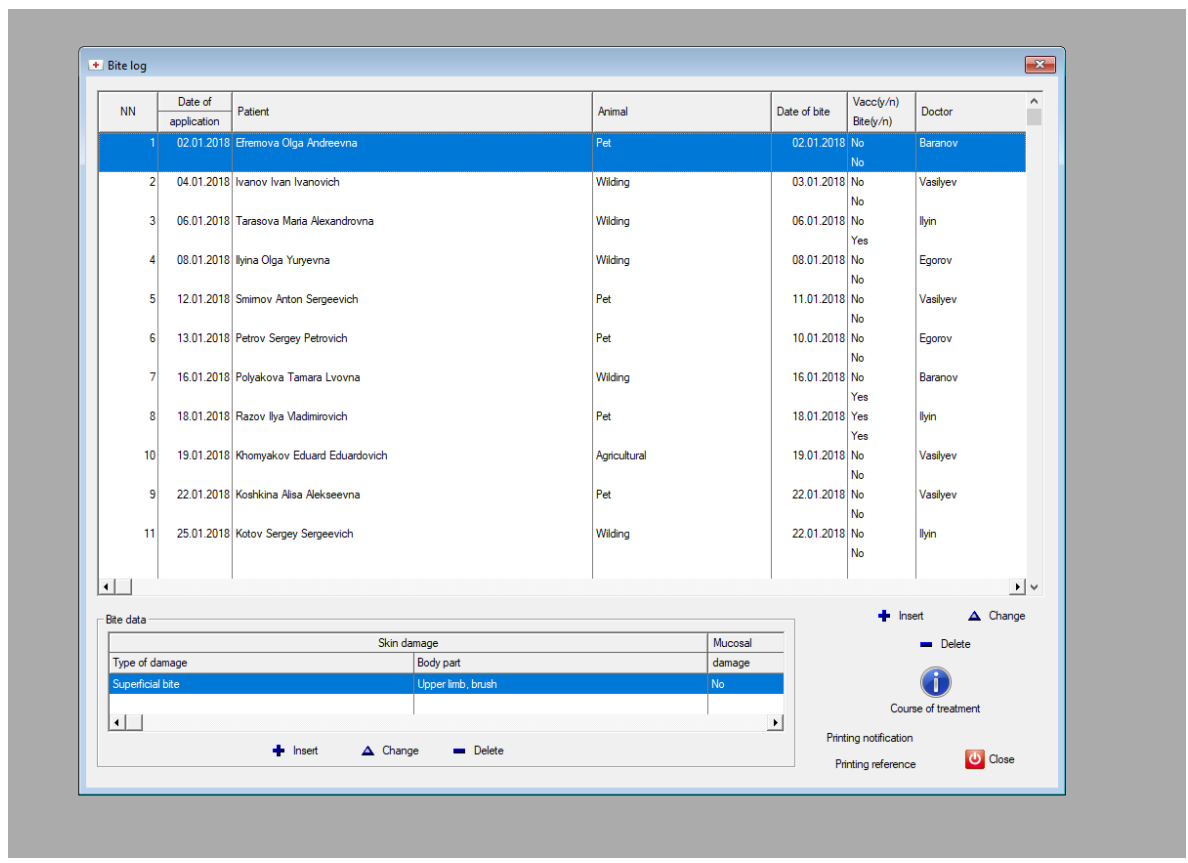

Fig. 2. Interface of the log of patients and their bites.

11) After each treatment event, the doctor makes a note in the schedule of vaccinations made for each patient. If the person does not appear on the appointed date for the next treatment action, the doctor uses the software to correct the previously drawn up plan of actions or makes a new one if the patient comes later.

12) If an animal that bites a person who has applied for anti-rabies help is under observation and has not died after 7 days, it means that it is healthy and the further vaccination against rabies is not required for the person it bites. Using the software product, you can delete actions that were fed into the database when creating a vaccination scheme, when they are not required. 
Implementation of AMDS in medical institutions of the Ministry of health of the Russian Federation will allow:

- to reduce the labor intensity and improve the efficiency of work of traumatologistsorthopedists on the formation of schedules of medical measures for patients in the framework of anti-rabies help to the population,

- to improve the efficiency of the analysis of the initial information about the patient's condition and the injuries to determine the category of contact with the animal and form a decision on anti-rabies measures,

- to automate the process of selecting vaccines and doses for patients of categories I, II, III of the depth of contact with animals when planning therapeutic measures of anti-rabies treatment,

- to automate the process of forming an action plan for anti-rabies prevention,

- to provide consulting assistance to the traumatologist-orthopedist in the formation of medical decisions for more complex cases of repeated visits, especially for patients of category III depth of contact with animals,

- to reduce the labor intensity of procedures for monitoring and correcting medical and preventive measures of the carried out anti-rabies assistance,

- to improve the efficiency of document management that accompanies the provision of anti-rabies care and prevention for the population, to speed up the search of information about previously treated patients and the formation of statistical reporting.

Consider in more detail the algorithm for making medical decisions when providing antirabies care. When performing treatment and prophylactic work after biting wild or domestic animals, it is necessary to take into account the importance of the criteria on the basis of which a medical decision is made. In our case, such criteria are:

K1 Full-course rabies vaccination over the past year

K2 Depth of contact with the animal, defined by the category

K3 The singular or not singular cases of animal bites over the past year

K4 General health of a patient affected by bites of an animal, its immune system

Table 1 will be used for the evaluation, indicating the wording of the different characteristics of the alternatives being compared.

Table 1. Intensity scale of relative importance.

\begin{tabular}{|c|c|}
\hline Relative importance intensity, $\beta_{\mathrm{i}}$ & Definition \\
\hline 1 & Equal importance \\
\hline 2 & Small superiority \\
\hline 3 & Moderate superiority \\
\hline 4 & Substantial superiority \\
\hline 5 & Significant superiority \\
\hline 6 & Very strong superiority \\
\hline
\end{tabular}

Next, experts build and fill in the matrix M, reflecting the results of a paired comparison of the criteria for forming a solution for providing anti-rabies assistance: 


$$
\left[\begin{array}{cccc}
K 1 & K 2 & K 3 & K 4 \\
K 2 & 1 & \beta_{2} & \beta_{3} \\
K 3 & 1 / \beta_{2} & 1 & \beta_{5} \\
K 4 & 1 / \beta_{3} & 1 / \beta_{5} & 1
\end{array}\right]
$$

In general, with $\mathrm{n}$ criteria compared, the matrix $\mathrm{M}$ has the following form:

$$
\mathrm{M}=\left[\begin{array}{ccc}
\mu_{11} & \cdots & \mu_{1 \mathrm{n}} \\
\cdots & \cdots & \cdots \\
\mu_{\mathrm{n} 1} & \cdots & \mu_{\mathrm{nn}}
\end{array}\right]
$$

Further processing includes calculating the local priority $\overrightarrow{\mathrm{P}}$. Based on the values of the elements of the $\mathrm{i}$-th term of the matrix $\mathrm{M}$, the $\mathrm{i}$-th coordinate of the vector $\overrightarrow{\mathrm{P}}$ is calculated: $\mathrm{p}_{\mathrm{i}}=\sqrt[\mathrm{n}]{\mu_{\mathrm{i} 1} \cdot \mu_{\mathrm{i} 2} \cdot \ldots \cdot \mu_{\mathrm{in}}}$, where $\mathrm{i}=\overline{1 . . \mathrm{n}}$. For further analysis, the vector $\overrightarrow{\mathrm{P}}$ normalizes, the normalized vector $\overrightarrow{\mathrm{P}^{*}}$ has the form: $\overrightarrow{\mathrm{P}^{*}}=\left(\begin{array}{c}\frac{\mathrm{p}_{1}}{\sum_{\mathrm{i}=1}^{\mathrm{n}} \mathrm{p}_{\mathrm{i}}} \\ \cdots \\ \frac{\mathrm{p}_{n}}{\sum_{\mathrm{i}=1}^{\mathrm{n}} \mathrm{p}_{\mathrm{i}}}\end{array}\right)$.

The analysis showed the following hierarchy of importance of criteria in prescribing therapeutic and preventive measures in the framework of assistance against rabies:

$1 \mathrm{~K} 2$ Depth of contact with the animal, defined by the category

$2 \mathrm{~K} 1$ Full-course rabies vaccination over the past year

$3 \mathrm{~K} 3$ The singular or not singular cases of animal bites over the past year

$4 \mathrm{~K} 4 \mathrm{General}$ health of a patient affected by bites of an animal, its immune system

In accordance with the priorities obtained, the depth of contact with the animal is first determined for treating rabies after bites of the animal. For this, the function $Y=d(x)$ is introduced, which as an argument receives a description of the nature of one injury received by patients from domestic or wild animals, and its values belong to the set $\mathrm{Y}=\{1,2,3\}$, the elements of which mean the first, second and third categories of contact with the animal. Such category values and description of the nature of the damage are defined in the antirabies instructions.

The definition area and the value area of the animal contact depth function $\mathrm{d}(\mathrm{x})$ are determined by table 2 .

Table 2. Definition area and the value area of the function d.

\begin{tabular}{|c|c|c|}
\hline $\begin{array}{c}\text { Numeric } \\
\text { value of } \\
\text { argument } \mathrm{x}\end{array}$ & Linguistic value of variable $\mathrm{x}$ & $\mathrm{Y}=\mathrm{d}(\mathrm{x})$ \\
\hline 0 & No skin damage & $\mathrm{d}(0)=1$ \\
\hline 1 & No skin and conjunctiva slobbering & $\mathrm{d}(1)=1$ \\
\hline 2 & Skin slobbering s in different places of the body & $\mathrm{d}(2)=2$ \\
\hline 3 & $\begin{array}{c}\text { Abrasions, superficial bites and scratches } \\
\text { of the body, upper and lower limbs }\end{array}$ & $\mathrm{d}(3)=2$ \\
\hline 4 & Conjunctiva slobbering & $\mathrm{d}(4)=3$ \\
\hline 5 & Head, neck, toes and toes bites & $\mathrm{d}(5)=3$ \\
\hline 6 & Deep wounds from pet attacks & $\mathrm{d}(6)=3$ \\
\hline 7 & Wounds from wild animal attacks & $\mathrm{d}(7)=3$ \\
\hline
\end{tabular}

For the functions of the depth of contact with the animal $d(x)$, the operation "generalization" (denoted by the sign "•") is proposed, which determines the category of 
injuries of the patient if the animal inflicted many injuries and bites on the patient. The generalization operation for functions $d(x)$ having different values takes on the value of most of the functions. If the generalization operation is applied to three or more functions $\mathrm{n}$, then the result of the operation will be the value that is present in the largest of the functions: $\mathrm{d}_{\Sigma}=\mathrm{d}_{1}(\mathrm{x}) \bullet \mathrm{d}_{2}(\mathrm{x}) \bullet \ldots \bullet \mathrm{d}_{\mathrm{n}}(\mathrm{x})=\underset{i=\frac{\max }{1 . . n}}{ }\left(d_{i}(x)\right)$.

Let $d_{1}(x)$ and $d_{2}(x)$ be functions, each of which describes a separate injury to a patient received from a wild or pet after its attack. To evaluate the two injuries received in the patient, a "generalization" operation should be applied, which will be set in the following table 3 .

Table 3. Generalization of the functions $d_{1}$ and $d_{2}$.

\begin{tabular}{|c|c|c|}
\hline $\mathrm{d}_{1}(\mathrm{x})$ & $\mathrm{d}_{2}(\mathrm{x})$ & $\mathrm{d}_{1}(\mathrm{x}) \bullet \mathrm{d}_{2}(\mathrm{x})$ \\
\hline 1 & 1 & 1 \\
\hline 1 & 2 & 2 \\
\hline 1 & 3 & 3 \\
\hline 2 & 2 & 2 \\
\hline 2 & 3 & 3 \\
\hline 3 & 3 & 3 \\
\hline
\end{tabular}

In the practice of medical workers, there are situations when, visually or on the basis of interviews with a patient, it is not always possible to unequivocally assess the extent of his damage after an animal bite, especially if the patient did not immediately seek medical help. Therefore, the depth of contact with the animal will be estimated considering the function of belonging $\mu_{\mathrm{P}}(\mathrm{x})$, with which it was possible to determine its damage after examining the patient.

In this case, an odd set of $\mathrm{P}$ is observed - "possible injuries of the patient", which, for example, may take the form: $\mathrm{P}=\{<2 ; 0.6>,<3 ; 0.8><5 ; 1>\}$. This set is defined on the universe $X=\{0,1,2,3,4,5,6,7\}$.

The set of values of the depth function of contact with an animal will also be an odd set, in the above example $\mathrm{Y}=\{<2 ; 0.6>,<2 ; 0.8><3 ; 1>\}$. To determine the category of contact with an animal in this case, the formula is used: $\mathrm{d}_{\Sigma}=\mathrm{d}_{1}(\mathrm{x}) \bullet \mathrm{d}_{2}(\mathrm{x}) \bullet \ldots \bullet \mathrm{d}_{\mathrm{n}}(\mathrm{x})=\underset{i=1 . . n}{\max }\left(d_{i}(x)\right)$, provided that the belonging function $\mu_{\mathrm{P}}(\mathrm{x}) \geq 0.6$.

After determining the category of contact with an animal, other criteria for forming a medical decision are also applied. It is determined whether the patient was previously vaccinated at least a year ago, animals are bitten for the first or not the first time during the year who applied for medical care. In view of the above, the decision tree is as follows, see figure 3 . 


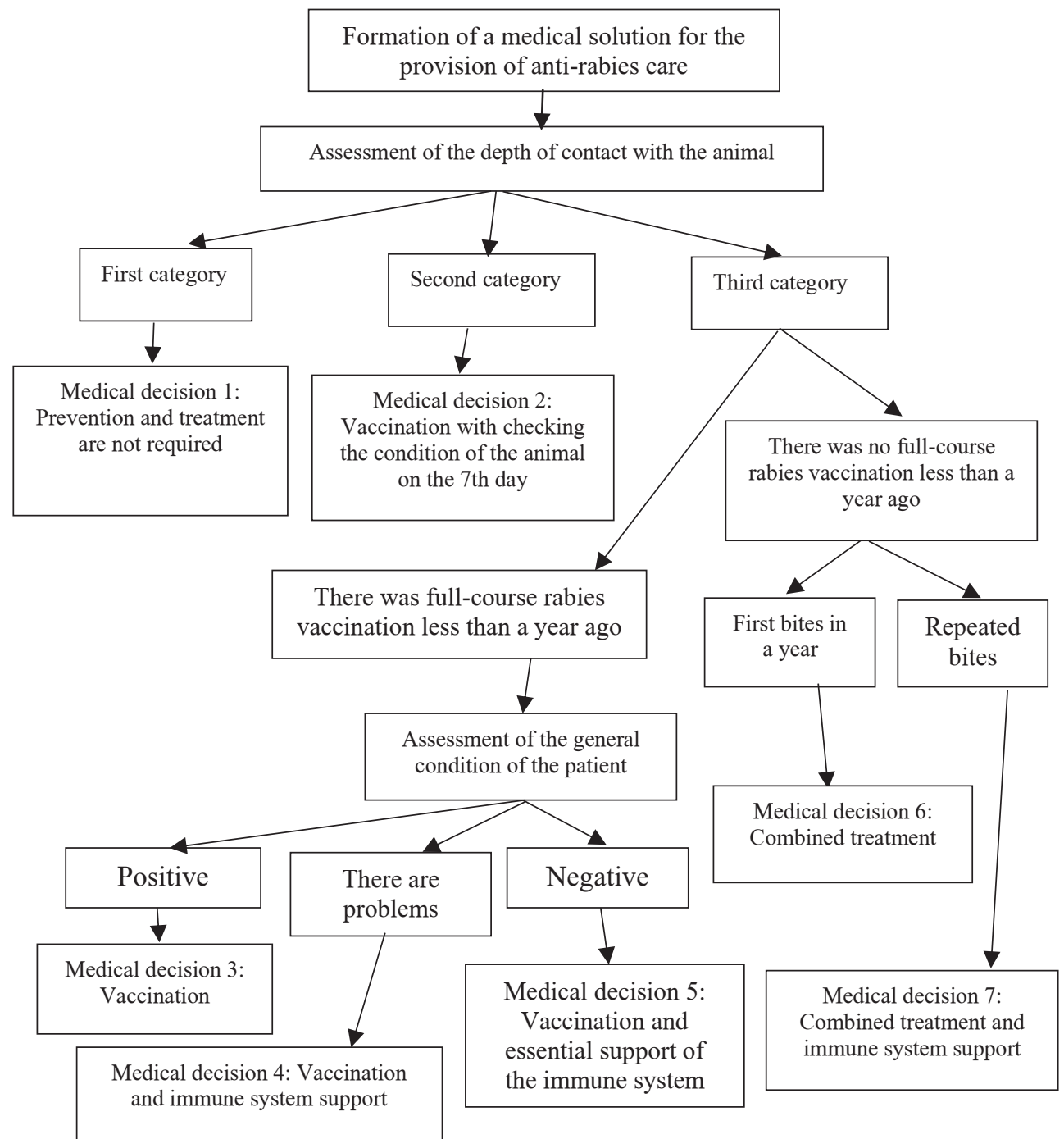

Fig. 3. Decision tree.

Above are considered only, decisions formed by the automated decision support system only at the stage of treatment prescribing. However, in the process of undergoing prevention and treatment against rabies due to the duration of this process, situations of violation of the treatment regimen arise, the automated system also has tools for correcting such situations.

\section{Discussions and conclusions}

During the research, a tool was developed to improve the scientific validity and accuracy of medical decisions on the issues of public immunization against rabies. The developed software product received the author's certificate no. 2018663452 dated 26.10.2018 from the Federal service for intellectual property (Rospatent). The software product has been tested in the emergency rooms of Moscow, in the practice of the Polyclinic Department of the First City hospital named after N. I. Pirogov. 
In addition to planning treatment and prevention measures, forming medical decisions within the framework of anti-rabies care and prevention, AMDSS can be used for scheduling treatment measures for various diseases that have some regularity, duration, and the final set of medications used, as well as for registering and monitoring their effectiveness.

\section{References}

1. Resolution of the Chief state sanitary doctor of the Russian Federation dated May 6, 2010 N 54 Moscow "About the approval of SP 3.1.7.2627-10" (in Russian)

2. The order of the Ministry of health of the Russian Federation dated 07.10.97 No. 297 "On improving the prevention of human infection with rabies" (together with "Provisions about the center of the anti-rabies aid in the subject of the Russian Federation", "Regulations about the order of work of medical institutions and centers") (in Russian)

3. Order of the Ministry of health of the Russian Federation dated March 21, 2014 No. 125n " On approval of the national calendar of preventive vaccinations and the calendar of preventive vaccinations for epidemic indications" (in Russian)

4. Rabies Sanitary rules SP 3.1.096-96, Veterinary rules VP 13.3.1103-96 https://rg.ru/2010/07/30/beshenstvo-dok.html (Last accessed 11.12.2020)

5. Instructions for COCAV use http://base.garant.ru/51521071 (Last accessed 14.12.2020)

6. D. Krenke, Theory and practice of database creating. 8th ed, 800, 166-195 (St. Petersburg, 2003)

7. E. A. Trachtengerts, Computer support for decision-making: a Scientific and practical publication. Series "Informatization of Russia on the threshold of the XXI century", 376, 53 (Moscow, SINTEG, 1998)

8. Prevention of infectious diseases. Prevention and control of infectious diseases common to humans and animals https://www.fsvps.ru/fsvps/laws/177.html (Last accessed 21.12.2020)

9. Rospotrebnadzor warned of high risks of rabies infection https://tass.ru/obschestvo/6721657 (Last accessed 21.12.2020)

10. F. Aggogeri, A. Borboni, A. Merlo, N. Pellegrini, R. Ricatto, Materials 10(3), art. no. 297 (2017) DOI: 10.3390/ma10030297 